\title{
Multivariate Texture-based Segmentation of Remotely Sensed Imagery for Extraction of Objects and Their Uncertainty
}

\author{
Arko Lucieer*, Alfred Stein* \& Peter Fisher** \\ * International Institute for Geo-Information Science and Earth Observation (ITC), \\ Department of Earth Observation Science, \\ P.O. Box 6, 7500 AA Enschede, the Netherlands
} Telephone: +31 (0)53 4874358, Fax: +31 (0)53 4874335 arko@lucieer.net stein@itc.nl ** University of Leicester, Department of Geography, Leicester, LE1 7RH, United Kingdom

Telephone: +44 (0)116 2523839, Fax: +44 (0)116 2523854 Email: pff1@le.ac.uk

Keywords: multivariate texture, image segmentation, Local Binary Pattern (LBP) operator, uncertainty

\begin{abstract}
In this study, a segmentation procedure is proposed based on grey-level and multivariate texture to extract spatial objects from an image scene. Object uncertainty was quantified to identify transitions zones of objects with indeterminate boundaries. The Local Binary Pattern
\end{abstract}


(LBP) operator, modeling texture, was integrated into a hierarchical splitting segmentation to identify homogeneous texture regions in an image. We proposed a multivariate extension of the standard univariate LBP operator to describe color texture. The paper is illustrated with two case studies. The first considers an image with a composite of texture regions. The two LBP operators provided good segmentation results on both grey-scale and color textures, depicted by accuracy values of $96 \%$ and $98 \%$ respectively. The second case study involved segmentation of coastal land cover objects from a multi-spectral Compact Airborne Spectral Imager (CASI) image, of a coastal area in the UK. Segmentation based on the univariate LBP measure provided unsatisfactory segmentation results from a single CASI band (70\% accuracy). A multivariate LBP based segmentation of three CASI bands improved segmentation results considerably (77\% accuracy). Uncertainty values for object building blocks provided valuable information for identification of object transition zones. We conclude that the (multivariate) LBP texture model in combination with a hierarchical splitting segmentation framework is suitable for identifying objects and for quantifying their uncertainty.

\section{Introduction}

Geospatial data quality is a topic frequently covered in recent scientific literature on GIS and remote sensing (Foody and Atkinson 2002). An important component of data quality is data uncertainty. Poor class definition, gradual transition zones or fuzzy boundaries, mixed pixels, and incomplete or imperfect data give rise to uncertainty in remotely sensed image classification results. Both fuzzy and probabilistic classification techniques can help to model and quantify uncertainty. In recent years, much research has focused on modeling uncertainty in remotely sensed image classification (Foody 1996, Hootsmans 1996, Canters 1997, Fisher 1999, van der Wel 2000, Zhang and Foody 2001, Foody and Atkinson 2002). It mainly 
focused on uncertainty of spectral classification on a pixel-by-pixel basis. As such, it partially ignored potentially useful spatial relations between pixels.

Object-oriented approaches to remotely sensed image processing have become popular with the growing amount of high-resolution satellite and airborne imagery. Segmentation techniques extract spatial objects from an image (Gorte and Stein 1998, Lucieer and Stein 2002). It extends classification, as spatial contiguity is an explicit goal of segmentation whereas it is only implicit in classification. Uncertainty in a segmented or classified image can affect further image processing. In particular, in areas where fuzzy objects or objects with indeterminate boundaries dominate, an indication of segmentation uncertainty is important.

A straightforward approach to identify fuzzy objects is to apply a fuzzy c-means (FCM) classification. This classifier gives membership values of belonging to each class for each pixel. A thematic map can be obtained from this result by labeling the pixels according to the class with the maximum membership value. However, pixel-based classifiers, like the FCM, do not take spatial relations between pixels into account, also known as pattern or texture. We argue that a texture-based segmentation approach (i.e. including the spatial component) can help to identify fuzzy objects. Texture reflects the spatial structure of pixel values and it is therefore indispensable in segmenting an area into sensible geographical units.

Texture analysis has been addressed and successfully applied in remote sensing studies in the past. An interesting overview paper concerning texture measures is Randen and Husøy (1999). Recently, Ojala and his co-workers have further pursued an efficient implementation and application towards texture-based segmentation (Ojala et al. 1996, 2002a; Ojala and 
Pietikäinen 1999; Pietikäinen et al. 2000). Their Local Binary Pattern (LBP) measure is superior to most of the traditional texture measures in segmentation of texture images (Ojala et al. 1996). LBP is a rotation invariant grey scale texture measure.

The aim of this study is to develop and apply a supervised multivariate texture segmentation technique to identify objects from remotely sensed imagery. It is applied to an image with a texture composition and to an airborne multispectral image of a coastal area in northwest England. It builds on work of Lucieer and Stein (2002) and Lucieer et al. (2004) and further explores the use of multivariate texture. In addition, we focus on quantification of object uncertainty to identify transition zones.

\section{Methods}

\subsection{Texture}

Image texture can provide valuable information for identification of objects. The human visual system not only can distinguish objects based on color, but texture plays an important role as well. A major characteristic of texture is the repetition of a pattern or patterns over a region. The pattern may be repeated exactly, or as a set of small variations, possibly as a function of position. There is also a random aspect to texture, because size, shape, color and orientation of pattern elements (sometimes called textons) can vary over a region.

A comparative study of texture measures is given in Randen and Husøy (1999). They conclude that a direction for future research is the development of powerful texture measures that can be extracted and classified with a low computational complexity. A relatively new and simple texture model is the Local Binary Pattern operator (LBP) (Pietikäinen et al. 2000, 
Ojala et al. 2002a). It is a theoretically simple yet efficient approach to grey scale and rotation invariant texture segmentation based on local binary patterns and non-parametric discrimination of sample and reference texture distributions.

\subsection{Texture model - the Local Binary Pattern Operator (LBP)}

Ojala et al. (2002a) derived the local binary pattern operator (LBP) by defining texture $T$ in a local neighborhood of a grey scale image as the joint distribution of grey levels of $P$ image pixels

$T=t\left(g_{c}, g_{0}, \ldots, g_{P-1}\right)$

where $g_{c}$ corresponds to the grey-scale value of the center pixel $\left(p_{c}\right)$ of the local neighborhood and $g_{i}(i=0, \ldots, P-1)$ corresponds to the grey-scale value of a pixel in the neighborhood of $p_{c}$. In this study, we apply a circle of radius $R$ with $P$ equally spaced pixels that form a circularly symmetric neighborhood set (Ojala et al. 2002a, Lucieer et al. 2004). A circular neighborhood enables a definition of a rotation invariant texture measure.

Invariance with respect to the scaling of pixel values or illumination differences is achieved by considering the signs of the differences instead of their numerical values

$T^{*} \approx t\left(\operatorname{sign}\left(g_{0}-g_{c}\right), \operatorname{sign}\left(g_{1}-g_{c}\right), \ldots, \operatorname{sign}\left(g_{P-1}-g_{c}\right)\right)$

Ojala et al. (2002a) found that not all local binary patterns describe properties of texture well. LBP captures the uniformity of the central pixel towards its neighborhood, but it does not 
capture the uniformity of the neighborhood itself. Therefore, they introduced a uniformity measure $U$ to define uniformity in a neighborhood set. $U$ corresponds to the number of spatial transitions or bitwise 0/1 changes in the pattern. With $g_{P}=g_{0}, U_{c}$ is defined as

$U_{C}=\sum_{i=1}^{P}\left|\operatorname{sign}\left(g_{i}-g_{c}\right)-\operatorname{sign}\left(g_{i-1}-g_{c}\right)\right|$

Patterns with $U_{c} \leq j$ are designated as uniform. Ojala et al. (2002a) found that for $j=2$ the best texture model is obtained for texture images. This results in the following operator for grey scale and rotation invariant texture description

$L B P_{c, j}= \begin{cases}\sum_{i=0}^{P-1} \operatorname{sign}\left(g_{i}-g_{c}\right) & \text { if } U_{C} \leq j \\ P+1 & \text { otherwise }\end{cases}$

The $L B P_{c, j}$ operator thresholds the pixels in a circular neighborhood of $P$ equally spaced pixels on a circle of radius $R$, at the value of the center pixel. It allows for detecting uniform patterns for any quantization of the angular space and for any spatial resolution. Non-uniform patterns are grouped under one label, $P+1$.

\subsection{A measure for texture}

The $L B P_{c, j}$ measures the spatial structure of local image texture, but discards contrast, being another important property of local image texture. In most cases, its performance can be enhanced by combining it with a rotation invariant variance measure that characterizes the contrast of local image texture, defined by 
$V A R_{c}=\sum_{i=0}^{P-1}\left(g_{i}-\mu_{c}\right)^{2}$, where $\mu_{c}=\frac{1}{P} \sum_{i=0}^{P-1} g_{i}$

$L B P_{c, j}$ and $V A R_{c}$ values are calculated and assigned to each individual image pixel, depicting local texture information. Therefore, two new images are derived from the original image containing $L B P_{c, j}$ and $V A R_{c}$ values for each pixel. These images form the basis for the final texture measure.

Most approaches to texture analysis quantify texture measures by single values (means, variances, entropy, etc.). However, much important information contained in the distributions of feature values might be lost. In this study, the final texture feature is the histogram of the joint $L B P_{c, j}$ and $V A R_{c}$ occurrence, computed over an image or a region of an image. The joint distribution of $\left(L B P_{c, j}, V A R_{c}\right)$ is approximated by a discrete two-dimensional histogram of size $P+2$ by $b$, where $P$ is the number of neighbors in a circular neighborhood and $b$ is the number of bins for $V A R_{c}$. The number of bins used in quantization of the feature space plays a crucial role. Histograms with too modest a number of bins fail to provide enough discriminative information about the distributions, however, if we go to the other extreme the number of entries per bin is very small and histograms become sparse and unstable. In this study following Ojala et al. (1996), the number of bins $b$ is computed by taking the total feature distribution of $\left(L B P_{c, j}, V A R_{c}\right)$ for the whole image. This distribution is divided into 32 bins having an equal number of entries. Ojala et al. (2002a) showed that the twodimensional $\left(L B P_{c, j}, V A R_{c}\right)$ histogram is a powerful tool for rotation invariant texture segmentation. 


\subsection{Texture similarity}

Similarity between different textures is evaluated as a test of goodness-of-fit using a nonparametric statistic, the log-likelihood ratio statistic, also known as the G-statistic (Sokal and Rohlf 1987). The G-statistic compares the bins of a texture sample histogram with a texture model histogram. The G-statistic is defined as

$$
G=2\left(\begin{array}{l}
{\left[\sum_{s, m} \sum_{i=1}^{t b} f_{i} \log f_{i}\right]-\left[\sum_{s, m}\left(\sum_{i=1}^{t b} f_{i}\right) \log \left(\sum_{i=1}^{t b} f_{i}\right)\right]-} \\
\left.\left[\sum_{i=1}^{t b}\left(\sum_{s, m} f_{i}\right) \log \left(\sum_{s, m} f_{i}\right)\right]+\left[\left(\sum_{s, m} \sum_{i=1}^{t b} f_{i}\right) \log \left(\sum_{s, m} \sum_{i=1}^{t b} f_{i}\right)\right]\right)
\end{array}\right)
$$

where, the sample $s$ is a histogram of the texture measure distribution of an image block, the model $m$ is a histogram of a reference area in the image of a particular texture, $t b$ is the total number of bins and $f_{i}$ is the probability for bin $i$. By using a nonparametric test we avoid making any, possibly erroneous, assumptions about the feature distributions. The value of the G-statistic indicates the probability that two sample distributions come from the same population: the higher the value, the lower the probability that the two samples are from the same population. The more alike the histograms are the smaller is the value of $G$.

Texture is modeled for certain image blocks. The block size should be appropriate for the computation of the texture features. As we consider blocks of increased size, however, the probability that regions contain a mixture of textures is increased. This can bias the comparison, since the reference textures contain only features of individual patterns. On the other hand, if the block size is too small it is impossible to calculate a texture measure. 
Within this constraint, it is impossible to define an optimum size for segmenting the entire image. Therefore, segmenting regions of a fixed block size is inappropriate (Aguado et al. 1998). Alternatively, a top-down hierarchical segmentation process, as discussed in the next section, offers a very suitable framework for segmenting image regions based on texture.

\subsection{Texture based image segmentation}

Split-and-merge segmentation consists of a region-splitting phase and an agglomerative clustering (merging) phase (Haralick and Shapiro 1985, Horowitz and Pavlidis 1976, Gorte and Stein 1998, Lucieer and Stein 2002, Lucieer et al. 2004). Supervised segmentation uses explicit knowledge about the study area to train the segmentation algorithm on reference textures. Aguado et al. (1998) introduced a segmentation framework with a top-down hierarchical splitting process based on minimizing uncertainty. In this study, we combine the $\left(L B P_{c, j}, V A R_{c}\right)$ texture measure and the segmentation framework as suggested by Aguado et al. (1998).

Similar to split-and-merge segmentation each square image block in the image is split into four sub-blocks forming a quadtree structure. The criterion used to determine if an image block is divided is based on a comparison between the uncertainty of the block and the uncertainty of the sub-blocks. The image is segmented such that uncertainty is minimized, where uncertainty is defined as the ratio between the similarity values (G-statistic), computed for an image block $B$, of the two most likely reference textures (equation 7). The reference textures are histograms of $\left(L B P_{c, j}, V A R_{c}\right)$ of characteristic regions in the image. To test for similarity between an image block texture and a reference texture, the $G$-statistic is applied. Uncertainty $U_{B}$ is then defined as 


$$
U_{B}=\frac{G_{1}}{G_{2}}
$$

where $G_{1}$ is the lowest $G$ value of all textures (highest similarity) and $G_{2}$ is the second lowest $G$ value. Uncertainty is high if $G_{1}$ and $G_{2}$ are very similar and $U_{B}$ is close to one. The subdivision of each image block is based on this uncertainty criterion. An image block is split into four sub-blocks if

$$
U_{B}>\frac{1}{4}\left(U_{S B 1}+U_{S B 2}+U_{S B 3}+U_{S B 4}\right)
$$

where the left side of equation 8 defines uncertainty obtained when the sub-blocks are labeled according to the reference class obtained by considering the whole block $(B)$. The right side of equation 8 defines uncertainty if the sub-blocks (SB1, SB2, SB3 and SB4) are labeled by the reference class obtained by the subdivision. Thus, the basic idea is to subdivide an image block only if it is composed of several textures. Additionally, segmentation is always uncertain at the boundaries, because the image block contains a mixture of textures. Accordingly, we subdivide blocks that have at least one neighboring region of a different texture (Aguado et al. 1998). Finally, we obtain a partition of the image. We consider an image object as a collection of contiguous image blocks sharing the same texture label.

The building blocks of each of the objects give information about object uncertainty. We use $U_{B}$ to depict uncertainty with which an object block is assigned a texture label. The spatial 
distribution of block uncertainty values within an object gives information about uncertainty of the spatial extent of objects. We expect high uncertainty values for the boundary blocks of objects, because of mixed textures and transition zones.

\subsection{Texture example}

Figure 1(a) shows a composite of five different textures. The image is derived from the Outex framework for testing texture models (Ojala et al. 2002b). These grey-scale textures were labeled with the following class names: class NW (granite), class NE (fabric), class SW (grass), class SE (stone) and class Center (reed mat). Each of these classes is unique in terms of texture. The image shows that the human visual system not only distinguishes image regions based on grey-scale or color, but also on texture, as one can clearly distinguish five homogeneous regions. A pixel-based classifier does not take into account texture or spatial information. Figure 1(b) shows why pixel-based classification techniques might fail. It shows the `defuzzified’ result of a pixel-based FCM classifier. In this case, a supervised fuzzy cmeans classification was applied with a Mahalanobis distance measure and an overlap parameter of 2.0 (Bezdek 1981, Zhang 2001). Five regions of 40 by 40 pixels were selected in the centers of the texture regions to train the classifier. Although, the patterns are still visible, no clear spatial partition of classes was obtained. A classification validation provided an overall accuracy of $30.00 \%$ and a Kappa coefficient of 0.13 .

\section{[FIGURE 1 ABOUT HERE]}

A much better segmentation was obtained when texture was incorporated by applying the unsupervised texture-based segmentation algorithm based on the joint $\left(L B P_{c, j}, V A R_{c}\right)$ 
distribution. A detailed description of these results can be found in Ojala and Pietikäinen (1999) and Lucieer et al. (2004). Additionally, a supervised approach might prove to be useful as one can guide the segmentation algorithm with reference texture information. Especially in geographical applications, a supervised approach is often feasible, as knowledge about the area might improve segmentation.

Figure 2 shows a supervised texture-based segmentation of figure 1(a), applying the uncertainty criteria of Aguado et al. (1998). Five references regions of 40 by 40 pixels were selected, corresponding to the five different textures in figure 1(a). Values for $P$ and $R$ were 8 and 1 respectively. An accuracy assessment of the segmentation results provided a very high overall accuracy of $96.20 \%$ and a Kappa coefficient of 0.95 , showing that good segmentation results can be obtained with the LBP texture measure. Uncertainty values were highest in class SW. This can be explained by the irregularity of this texture, i.e. its pattern is not repetitive and the reference area does not fully represent the whole texture area. In addition, all small blocks at the boundaries of textures show high $(>0.9)$ uncertainty values, because they contain mixtures of different textures.

[FIGURE 2 ABOUT HERE]

\subsection{A multivariate texture model}

The $L B P_{c, j}$ texture measure allows a texture description of a single band. Most remote sensing images, however, consist of multiple bands. Including multiple bands might improve segmentation considerably, as a combination of bands provides more spectral information for identification of different land cover types. 
In their psychophysical study Poirson and Wandell (1996) showed that color and pattern information are processed separately by the human visual system. Mojsilovic et al. (2000) extracted color-based information from the luminance and chrominance color components. The achromatic pattern component was utilized as texture pattern information. Another approach was that of Panjwani and Healey (1995) which captured spatial interactions both within and between color bands with Markov random fields (MRFs). More recently, Pietikäinen et al. (2002) showed that the powerful LBP texture measure can also be applied to color images. They processed color information and texture information separately and obtained good classification results for color texture images.

Most research on color texture focused on images of different materials with a clear texture. In standard color images, the pattern in different bands is often highly correlated. This makes it possible to summarize pattern information in a single band and process it separately from color information. In remote sensing images, however, information is recorded from different parts of the spectrum. Therefore, textures in these bands are not necessarily similar. In between band relations should be taken into account when looking at multivariate texture measures for remotely sensed imagery. The $L B P_{c, j}$ texture measure is a robust, rotation invariant and flexible texture measure. An extension to the multivariate case is expected to provide good segmentation results.

In this study, a new multivariate texture measure is introduced and implemented. It is based on the univariate $L B P_{c, j}$ measure. The Multivariate Local Binary Pattern operator, $M L B P_{c}$ describes local pixel relations in three bands. In addition to the spatial interactions of pixels 
within one band, interactions between bands are considered. Thus, the neighborhood set for a pixel consists of the local neighbors in all three bands (figure 3). The local threshold is taken from these bands, which makes up a total of nine different combinations. This results in the following operator for a local color texture description

$$
\begin{gathered}
\operatorname{sign}\left(g_{i}^{b 1}-g_{c}^{b 1}\right)+\operatorname{sign}\left(g_{i}^{b 2}-g_{c}^{b 1}\right)+\operatorname{sign}\left(g_{i}^{b 3}-g_{c}^{b 1}\right)+ \\
M L B P_{c}=\sum_{i=0}^{P-1} \operatorname{sign}\left(g_{i}^{b 1}-g_{c}^{b 2}\right)+\operatorname{sign}\left(g_{i}^{b 2}-g_{c}^{b 2}\right)+\operatorname{sign}\left(g_{i}^{b 3}-g_{c}^{b 2}\right)+ \\
\operatorname{sign}\left(g_{i}^{b 1}-g_{c}^{b 3}\right)+\operatorname{sign}\left(g_{i}^{b 2}-g_{c}^{b 3}\right)+\operatorname{sign}\left(g_{i}^{b 3}-g_{c}^{b 3}\right)
\end{gathered}
$$

where $b 1$ is the first band, $b 2$ is the second band, and $b 3$ is the third band. The first part of the equation calculates LBP values for the center pixel of the first band based on relations with the neighbors in the first band and the two other bands. The second part of the equation calculates LBP values for the center pixel of the second band and the third part of equation 9 calculates LBP values for the center pixel of the third band. Each of the three central pixels is, therefore, compared with neighborhood pixels in the other bands. $M L B P_{c}$ is not just a summation of $L B P_{c, j}$ of individual bands, it also models pixel relations between bands. These cross-relations can be important in the distinction of different color textures. A total of nine LBP values is obtained and summed to derive $M L B P_{c}$. The color texture measure is the histogram of $M L B P_{c}$ occurrence, computed over an image or a region of an image. This single distribution contains $P \times 3^{2}$ bins (e.g. $P=8$ results in 72 bins).

[FIGURE 3 ABOUT HERE]

$M L B P_{c}$ measures the binary color pattern of a texture. To complete this measure with contrast and variance information we included the color histogram, $R G B-3 D$. Each 8-bit band 
is quantized into 32 levels by dividing the pixel values on each band by 8 , resulting in a three-dimensional histogram with $32^{3}$ entries. The two histograms of $M L B P_{c}$ and $R G B-3 D$ are used to segment a three-band image into objects. In the top-down hierarchical splitting process we calculate $M L B P_{c}$ and $R G B$-3D histograms for every image block. G-statistic values are calculated to test for similarity between image block and reference texture histograms. For two $M L B P_{c}$ and $R G B-3 D$ histograms, two G-statistic values are obtained. These values are summed to derive a single similarity measure. Based on this measure, uncertainty values are calculated using equation 7 and texture labels are assigned to image blocks to form objects

To illustrate the solution for segmenting regions of different color texture, a three-band image (512 by 512 pixels) with a composition of six different color textures (figure 4(a)) was used. This image was composed of textures from the Outex texture library (Ojala et al., 2002b). The following textures were used: Upper Left (UL) = fur, Upper Right (UR) = carpet, Middle Left $(\mathrm{ML})=$ wood, Middle Right $(\mathrm{MR})=$ pasta, Lower Right $(\mathrm{LR})=$ flour, Lower Left $(L L)=$ seeds. It poses a more difficult segmentation problem than the grey-scale texture composition of figure 1(a), because of the high variance in color and the different texture scales. Six references regions of 40 by 40 pixels were selected, corresponding to the six different texture classes. Values for $P$ and $R$ were 8 and 1 respectively. Figure 4(b) shows the segmentation result. All regions were identified correctly. In the lower left object (LL), however, some dark spots were (incorrectly) segmented as fur (UL). This was most likely caused by high similarity in color distributions. Additionally, in the lower right object (LR) some dark shadow spots were (incorrectly) segmented as flour (LL). Uncertainty for these incorrectly labeled objects and boundary regions was high (>0.9) (figure 4(c)). 
An accuracy assessment of the segmentation result provided an overall accuracy of $98.32 \%$ and a Kappa coefficient of 0.98. The confusion matrix with per-texture accuracy percentages is given in table 1 . These accuracy values show that good segmentation results can be obtained with the multivariate LBP texture measure.

[FIGURE 4 ABOUT HERE]

[TABLE 1 ABOUT HERE]

\section{Case study}

\subsection{Study area: the Ainsdale Sands}

The study area, known as the Ainsdale Sands, is on the coast of Northwest England approximately 25km North of Liverpool. The Ainsdale Sand Dunes National Nature Reserve (NNR) totals 508 ha and forms part of the Sefton Coast. The NNR is within the coastal Special Protection Area. It contains a range of habitats, including intertidal sand flats, embryo dunes, high mobile yellow dunes, fixed vegetated dunes, wet dune slacks, areas of deciduous scrub and a predominantly pine woodland. Management of this area consists of extending the area of open dune habitat through the removal of pine plantation from the seaward edge of the NNR, maintaining and extending the area of fixed open dune by grazing and progressively creating a more diverse structure within the remaining pine plantation with associated benefits for wildlife (Sefton Coast Partnership, 2004).

In 1999, 2000 and 2001 the Environment Agency, UK, collected fine spatial resolution 
digital surface models (DSM) by LiDAR, and simultaneously, acquired multi-spectral Compact Airborne Spectral Imager (CASI) imagery (one flight each year). The aircraft flew at approximately $800 \mathrm{~m}$ above ground level, acquiring $2 \mathrm{~m}$ spatial resolution LiDAR scenes and $1 \mathrm{~m}$ spatial resolution CASI imagery. In this study, the CASI image of 2001 was used. These images, geometrically corrected by the Environment Agency, were spatial composites of multiple flight strips. The area covered by these images was approximately $6 \mathrm{~km} 2$.

We applied the univariate segmentation algorithm on the LiDAR DSM to derive general landform classes (Lucieer et al., 2004). An accuracy assessment of the segmentation results provided an overall accuracy of $86 \%$. The results showed that the univariate LBP measure in combination with the hierarchical splitting algorithm can provide a meaningful segmentation of basic land form classes with an indication of object uncertainty. In this study, we focus on segmentation of land cover classes from multispectral CASI imagery.

\subsection{Land cover segmentation}

Land cover is obtained from spectral information from the CASI image. Four land cover classes can be distinguished: sand, marram grass, willow shrub and woodland. Detailed mapping of these units is required, because knowledge about the location and dynamics of these object types is important for monitoring the rare habitats in this area, as well as, the coastal defense against flooding.

Figure 5(a) shows a subset (512 by 512 pixels) of band 12 of the CASI image of the study area. Band 12 at $780 \mathrm{~nm}$ (NIR) was chosen for a univariate segmentation based on the joint distribution of $L B P_{c, j}$ and $V A R_{c}$ values. It is suitable for discrimination of land cover types, 
because of large differences in reflectance for different vegetation types. Four reference areas of 40 by 40 pixels were selected to train the algorithm. Values for $P$ and $R$ were 8 and 1 respectively. Figure 6(a) shows a segmentation of band 12 with the $\left(L B P_{c, j}, V A R_{c}\right)$ texture measure for four land cover classes. The woodland area in the southeast corner of the image was correctly segmented with uncertainty values between 0.1 and 0.5 (figure 6(b)). The northeastern corner of the image and small objects in the northern part of the image were also segmented as woodland. However, fieldwork showed that no woodland occurred in this area.

The main part of the dune field was segmented as willow shrub land. Fieldwork showed that marram grass was mainly found on the fore dune and on the highest parts of the dune ridges in the dune field. Only a few small patches of marram grass can be seen in figure 6(a) in the fore dune area. Willow shrub was found all over the dune field, but mainly in the dune slacks. Image texture for these two classes, however, is very similar in band 12 of the CASI image. High uncertainty values (higher than 0.7 in the dune field and higher than 0.95 in the fore dune and dune ridge areas) in figure 6(b) confirm the similarity of these two land cover classes. The sand on the beach was correctly segmented, because of its characteristic texture. Uncertainty values were lower than 0.2 in this area. Again, a short transition zone can be seen from the fore dune to the beach with decreasing marram grass coverage (figure 6(b)). This zone is depicted by uncertainty values of 0.95 and higher. Field observations showed that the univariate texture-based segmentation algorithm performed unsatisfactorily, especially in areas where marram grass was severely under-segmented. Table 2 confirms this observation, showing accuracy values for individual land cover classes. The overall segmentation accuracy was $70.53 \%$ and the Kappa coefficient was 0.61 . It can be concluded from table 2 that major marram grass areas were incorrectly segmented as willow shrub. 
[FIGURE 6 ABOUT HERE]

[TABLE 2 ABOUT HERE]

\subsection{Multivariate texture-based land cover segmentation}

Segmentation using only one CASI band discards valuable information in other bands. A multivariate approach towards texture segmentation might improve segmentation results. The combined $M L B P_{c}$ and $R G B-3 D$ texture measure, models texture in three bands. CASI band 1 (440 nm), $8(650 \mathrm{~nm})$ and $12(780 \mathrm{~nm})$ explain most of the variance in the image scene and characterize land cover classes well. Figure 5(b) shows a color composite of these three bands. Figure 7 shows a supervised segmentation based on $M L B P_{c}$ and $R G B-3 D$. Segmentation of the marram grass class improved considerably. The fore dune area and the dune ridges were segmented as marram grass, as was observed in the field. The core areas showed low uncertainty values, whereas the boundaries showed high uncertainty values. This corresponds to observations that marram grass gradually changed to willow shrub land in the dune slacks and to sandier terrain towards the beach side. The woodland area was segmented correctly. In addition, segmentation of the north-eastern part of the area (marram grass and willow shrub) improved, as the segmentation result of a single band (figure 6) showed woodland in this area. The beach area was correctly segmented with low uncertainty values. Some small incorrectly segmented blocks (marram, willow and woodland) occurred in the beach area where the sand was wet with low reflectance values in the image. High uncertainty values $(>0.9)$ occurred in all transition areas. These uncertainty values are an indication for the occurrence of fuzzy objects with indeterminate boundaries. 
An accuracy assessment of the segmentation results provided an overall accuracy of $77.09 \%$ and a Kappa coefficient of 0.71 . The confusion matrix with per-class accuracy percentages is given in table 3. It can be concluded from this confusion matrix that segmentation of marram grass and willow shrub improved considerably compared to segmentation based on one CASI band.

[FIGURE 7 ABOUT HERE]

[TABLE 3 ABOUT HERE]

\section{Discussion and conclusions}

In this study, a texture-based supervised segmentation algorithm derived labeled objects from remotely sensed imagery. Texture was modeled with the joint distribution of the local binary pattern (LBP) operator and local variance. The segmentation algorithm was based on a hierarchical splitting technique, reducing uncertainty at the level of the image blocks that were obtained. By applying this technique, one does not only obtain a texture-based image segmentation, yet also an indication of uncertainty for all object building blocks. The spatial distribution of uncertainty values provided information about the location and width of transition zones.

The univariate $L B P_{c, j}$ texture measure was extended to a multivariate measure, $M L B P_{c}$, to model within band and between band pixel relations in three bands. The $M L B P_{c}$ measure was further extended with color information using a three-dimensional color histogram, $R G B-3 D$. The combination of these texture measures, model color texture as registered on different 
bands.

The univariate LBP measure provided good segmentation results for a test case study with a composite image of five different grey-scale textures. An overall accuracy of $96 \%$ was obtained. An artificial image with a composition of six color textures was used to demonstrate the use of $M L B P_{c}$ and $R G B-3 D$ in segmentation. Good segmentation results were obtained from this complex texture image, depicted by an overall accuracy of $98 \%$.

To illustrate the algorithm for mapping coastal objects, a CASI image of a coastal area on the northwest coast of England was used. Land cover objects derived from band 12 of the CASI image showed high uncertainty values and many incorrectly labeled objects. The overall accuracy was 71\%. Additionally, compared to field observations segmentation results were unsatisfactorily. The combination of textural and spectral information from more than one CASI band greatly improved segmentation results. The $M L B P_{c}$ and $R G B-3 D$ based segmentation was applied to band 12, 8 and 1 of the CASI image of the study area. Segmentation results improved considerably, depicted by and overall accuracy of $77 \%$. Uncertainty values provided valuable information about transition zones between fuzzy objects.

In this study, we applied a texture-based segmentation algorithm on airborne imagery for identification of coastal objects. The proposed algorithm, however, can easily be applied to other remote sensing images and other study areas. The univariate and multivariate LBP measures can also be used in a different context. Contextual classification using the LBP 
texture measure might provide valuable results from image classification. The computation of the multivariate LBP measure was limited to three bands. More image bands could be used in the $M L B P_{c}$ and $R G B-3 D$ texture model. It would, however, increase complexity and computational demands considerably, whereas extra bands would possibly not add much textural information. For multispectral and hyperspectral images, one could include a preprocessing step to detect the three bands that explain most variance for a specific application

In this study, a uniformity measure is defined for the univariate LBP measure. It depicts uniformity of pixel values in a neighborhood set. Ojala et al. (2002) showed that more than $90 \%$ of the patterns in a texture image are uniform. In remote sensing images, however, also non-uniform patterns occur. Some of these non-uniform patterns might be characteristic for a certain land cover class. An extension of the uniformity measure to the multivariate case might provide more information on pattern uniformity in remotely sensed imagery. A multivariate uniformity measure could be calculated by summation of uniformity in each band or by combining the uniformity measure for each of the nine components in the multivariate LBP measure (equation 9). We will assess the effect of a multivariate uniformity measure on segmentation of multispectral remote sensing imagery in future research.

The resolution of the neighborhood set applied during segmentation affects the texture measure and, therefore, the segmentation result. In this study, a (circular) neighborhood set of the nearest eight neighboring pixels was used. Circular neighborhood sets with large radii and a large number of neighbors might improve description of large-scale textures. Therefore, a multi-resolution approach with different combinations of neighborhood sets might provide a meaningful texture description. In future research, the effect of different 
neighborhood sets on the segmentation result will be assessed.

\section{References}

AGUADO, A.S., MONTIEL, E., AND NIXON, M.S., 1998, Fuzzy image segmentation via texture density histograms. EU project Nr. ENV4-CT96-0305 - Fuzzy Land Information from Environmental Remote Sensing (FLIERS) Final Report, 1998.

BEZDEK, J., 1981, Pattern Recognition with Fuzzy Objective Function Algorithms (Plenum Press, New York).

CANTERS, F., 1997, Evaluating the uncertainty of area estimates derived from fuzzy land cover classification. Photogrammetric Engineering \& Remote Sensing, 63, 403-414.

CHENG, T., FISHER, P.F., AND ROGERS, P., 2002, Fuzziness in multi-scale fuzzy assignment of duneness. Accuracy 2002 - International Symposium On Spatial Accuracy Assessment in Natural Resources and Environmental Sciences, pp.154-159.

CHENG, T. AND MOLENAAR, M., 2001, Formalizing fuzzy objects from uncertain classification results. International Journal of Geographical Information Science, 15, 27-42.

FISHER, P.F., 1999, Models of Uncertainty in Spatial Data, In Geographical Information Systems, second edition (Wiley \& Sons, New York), pp. 191-205.

FISHER, P., CHENG, T. AND WOOD, J., 2004, Where is Helvellyn? Multiscale morphometry and the mountains of the English lake district, Transactions of the Institute of British Geographers, 29, 106-128.

FOODY, G. M., 1996, Approaches for the production and evaluation of fuzzy land cover classifications from remotely sensed data. International Journal of Remote Sensing, 17, 13171340.

FOODY, G.M. AND ATKINSON, P.M., 2002, Uncertainty in GIS and Remote Sensing (John Wiley \& Sons Ltd). 
GORTE, B. H. H. AND STEIN, A., 1998, Bayesian classification and class area estimation of satellite images using stratification. IEEE Transactions on Geosciences and Remote Sensing, 36, 803-812.

HARALICK, R.M., SHANMUGAM, K., AND DINSTEIN, I., 1973, Textural features for image classification. IEEE Transactions on Systems, Man and Cybernetics, 2, 610-621.

HARALICK, R.M. AND SHAPIRO, L.G., 1985, Image segmentation techniques. Computer Vision, Graphics and Image Processing, 29, 100-132.

HOOTSMANS, R.M., 1996, Fuzzy Sets and Series analysis for Visual Decision Support in Spatial Data Exploration. PhD thesis, Utrecht University.

HOROWITZ, S.L. AND PAVLIDIS, T., 1976, Picture segmentation by a tree traversal algorithm. Journal of the Association for Computing Machinery, 23, 368-388.

LUCIEER, A. AND STEIN, A., 2002, Existential uncertainty of spatial objects segmented from remotely sensed imagery. IEEE Transactions on Geoscience and Remote Sensing, 40, 2518- 2521.

LUCIEER, A., FISHER, P. AND STEIN, A., 2004, GeoDynamics (CRC Press LLC), chapter Texture-based Segmentation of Remotely Sensed Imagery to Identify Fuzzy Coastal Objects. MOJSILOVIC, A., KOVACEVIC, J., HU, J., SAFRANEK, R. AND GANAPATHY, S., 2000, Matching and retrieval based on the vocabulary and grammar of color patterns, IEEE Transactions on Image Processing, 9, 38-54.

NIXON, M.S. AND AGUADO, A.S., 2002, Feature extraction \& image processing (Butterworth-Heinemann).

PANJWANI, D. AND HEALEY, G., 1995, Markov random field models for unsupervised segmentation of textured color images, IEEE Transactions on Pattern Analysis and Machine Intelligence, 17, 939-954.

PIETIKÄINEN, M., MÄENPÄÄ, T. AND VIERTOLA, J., 2002, Color texture classification 
with color histograms and local binary patterns, Proceedings of the Second International Workshop on Texture Analysis and Synthesis, Copenhagen, Denmark, 109-112.

POIRSON, B. AND WANDELL, B., 1996, Pattern-color separable pathways predict sensitivity to simple colored patterns, Vision Research 36, 515-526.

OJALA, T., PIETIKÄINEN M. AND HARWOOD, D., 1996, A comparative study of texture measures with classification based on feature distributions. Pattern Recognition, 29, 51-59.

OJALA, T. AND PIETIKÄINEN, M., 1999, Unsupervised texture segmentation using feature distributions. Pattern Recognition, 32, 477-486.

OJALA T., PIETIKÄINEN M., AND MÄENPÄÄ, T., 2002a, Multiresolution gray-scale and rotation invariant texture classification with local binary patterns. IEEE Transactions on Pattern Analysis and Machine Intelligence, 24, 971-987.

OJALA, T., MÄENPÄÄ, T., PIETIKÄINEN, M., VIERTOLA, J., KYLLÖNEN, J. AND HUOVINEN, S., 2002b, Outex - New framework for empirical evaluation of texture analysis algorithms. 16th International Conference on Pattern Recognition, Quebec, Canada, 1:701706. URL: http://www.outex.oulu.fi

PIETIKÄINEN M., OJALA T., AND XU, Z., 2000, Rotation-invariant texture classification using feature distributions. Pattern Recognition, 33, 43-52.

RANDEN, T. AND HUSØY, J.H., 1999, Filtering for Texture Classification: A Comparative Study. IEEE Transactions on Pattern Analysis and Machine Intelligence, 21, 291-310.

SEFTEN COAST PARTNERSHIP, 2004, Sefton coast partnership — nature conservation, URL: http://www.seftoncoast.org.uk

SOKAL, R.R. AND ROHLF, F.J., 1987, Introduction to Biostatistics, second edition (W.H. Freeman and Co, New York).

WEL, F. VAN DER, 2000, Assessment and Visualisation of Uncertainty in Remote Sensing Land Cover Classifications. PhD thesis, Utrecht University. 
ZHANG J. AND FOODY, G. M., 2001, Fully-fuzzy supervised classification of sub-urban land cover from remotely sensed imagery: Statistical and artificial neural network approaches. International Journal of Remote Sensing, 22, 615-628. 


\section{Tables}

Table 1. Confusion matrix with accuracy values (\%) of a multivariate texture-based segmentation. Overall accuracy was $98.32 \%$ and the Kappa coefficient was 0.98 .

Table 2. Confusion matrix with per-class accuracy values (\%) of a univariate texture-based segmentation of band 12 of the CASI image. Overall accuracy was $70.53 \%$ and the Kappa coefficient was 0.61 .

Table 3. Confusion matrix with per-class accuracy values (\%) of a (multivariate) texturebased segmentation of bands 12, 8 and 1 of the CASI image. Overall accuracy was 77.09\% and the Kappa coefficient was 0.71 .

Table 1

\begin{tabular}{|c|ccccccc|}
\hline & \multicolumn{7}{|c|}{ Reference } \\
\hline Class & UL & UR & ML & MR & LL & LR & Total \\
\hline UL & 99.93 & 0.56 & 1.31 & 0.26 & 1.91 & 0.38 & 12.72 \\
UR & 0.02 & 99.41 & 0.03 & 1.59 & 0.02 & 0.02 & 24.11 \\
ML & 0.05 & 0.00 & 98.09 & 0.05 & 0.12 & 0.21 & 9.62 \\
MR & 0.00 & 0.02 & 0.02 & 97.82 & 0.02 & 0.09 & 16.44 \\
LL & 0.00 & 0.01 & 0.55 & 0.10 & 97.68 & 2.30 & 21.77 \\
LR & 0.00 & 0.00 & 0.00 & 0.16 & 0.26 & 97.00 & 15.35 \\
Total & 100.00 & 100.00 & 100.00 & 100.00 & 100.00 & 100.00 & 100.00 \\
\hline
\end{tabular}

Table 2

\begin{tabular}{|c|ccccc|}
\hline & \multicolumn{5}{|c|}{ Reference } \\
\hline Class & Sand & Marram Grass & Willow Shrub & Woodland & Total \\
\hline Sand & 100.00 & 0.00 & 0.00 & 0.00 & 26.42 \\
Marram Grass & 0.00 & 0.00 & 0.22 & 0.00 & 0.06 \\
Willow Shrub & 0.00 & 98.94 & 99.78 & 0.00 & 41.99 \\
Woodland & 0.00 & 1.06 & 0.00 & 100.00 & 31.54 \\
Total & 100.00 & 100.00 & 100.00 & 100.00 & 100.00 \\
\hline
\end{tabular}

Table 3

\begin{tabular}{|c|ccccc|}
\hline & \multicolumn{5}{|c|}{ Reference } \\
\hline Class & Sand & Marram Grass & Willow Shrub & Woodland & Total \\
\hline Sand & 100.00 & 0.00 & 2.38 & 0.00 & 26.96 \\
Marram Grass & 0.00 & 96.92 & 20.29 & 0.00 & 20.98 \\
Willow Shrub & 0.00 & 2.11 & 64.77 & 0.00 & 17.34 \\
Woodland & 0.00 & 0.97 & 12.55 & 100.00 & 34.73 \\
Total & 100.00 & 100.00 & 100.00 & 100.00 & 100.00 \\
\hline
\end{tabular}




\section{Figure Captions}

Figure 1. Texture image composition: (a) artificial composition of five different natural textures with five reference areas (Ojala et al. 2002b); (b) result of a pixel-based classifier. Figure 2. Supervised texture-based segmentation: (a) segmentation based on the joint $L B P_{c, j}$ and $V A R_{c}$ distribution with five reference classes; (b) related uncertainty for all object building blocks.

Figure 3. The neighborhood set for the multivariate (three band) LBP texture measure describes spatial pixel relations within a band and between bands.

Figure 4. Segmentation of color texture image: (a) artificial composition of five different color textures (Ojala et al. 2002b); (b) supervised texture-based segmentation based on the multivariate $M L B P_{c}$ distribution and $R G B-3 D$ color histogram with five reference classes; (c) related uncertainty for all object building blocks.

Figure 5. CASI image of the Ainsdale sands study area, UK: (a) band 12; (b) color composite of band 12, 8 and 1 (RGB).

Figure 6. Segmentation of land cover from CASI image: (a) supervised texture-based segmentation of band 12 (NIR) of the CASI image with four reference land cover classes, based on the joint univariate $L B P_{c, j}$ and $V A R_{c}$ distribution; (b) related uncertainty for all object building blocks.

Figure 7. Multivariate texture-based segmentation of land cover from CASI image: (a) supervised segmentation of band 12, 8 and 1 based on $M L B P_{c}$ distribution and $R G B-3 D$ color histogram; (b) related uncertainty for all object building blocks. 
Figures

Figure 1

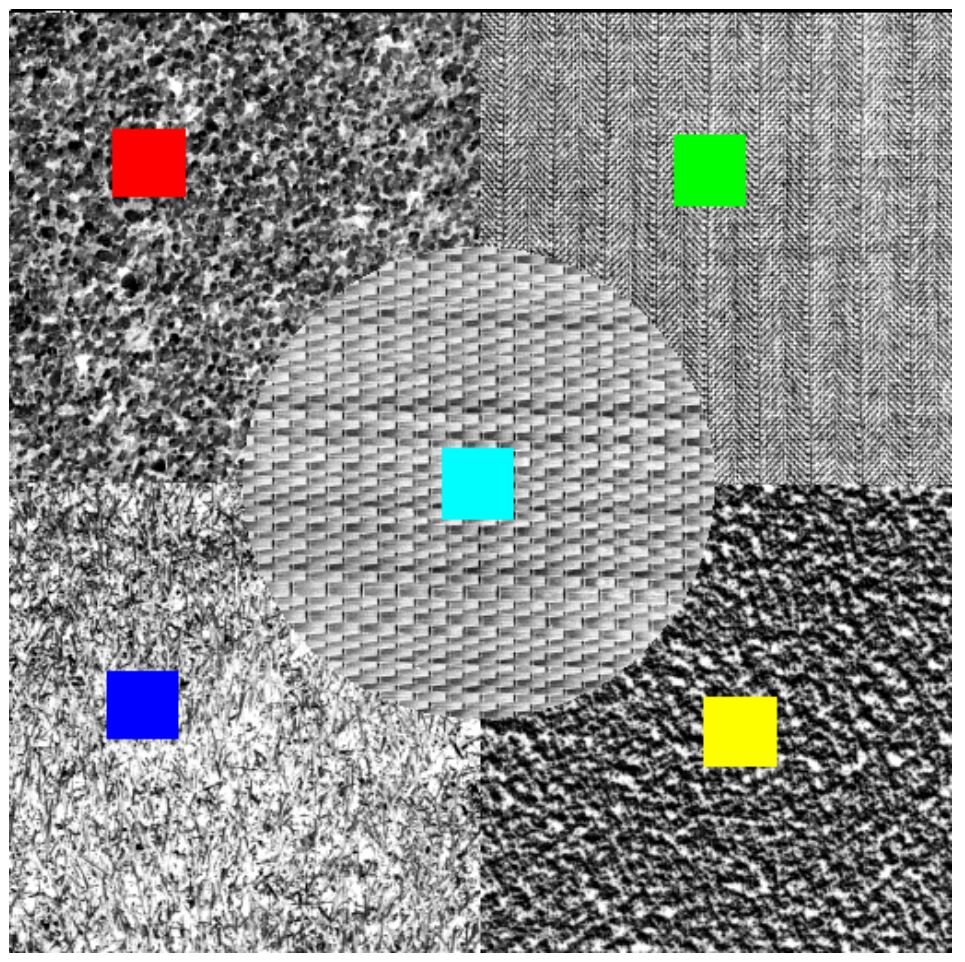

(a)

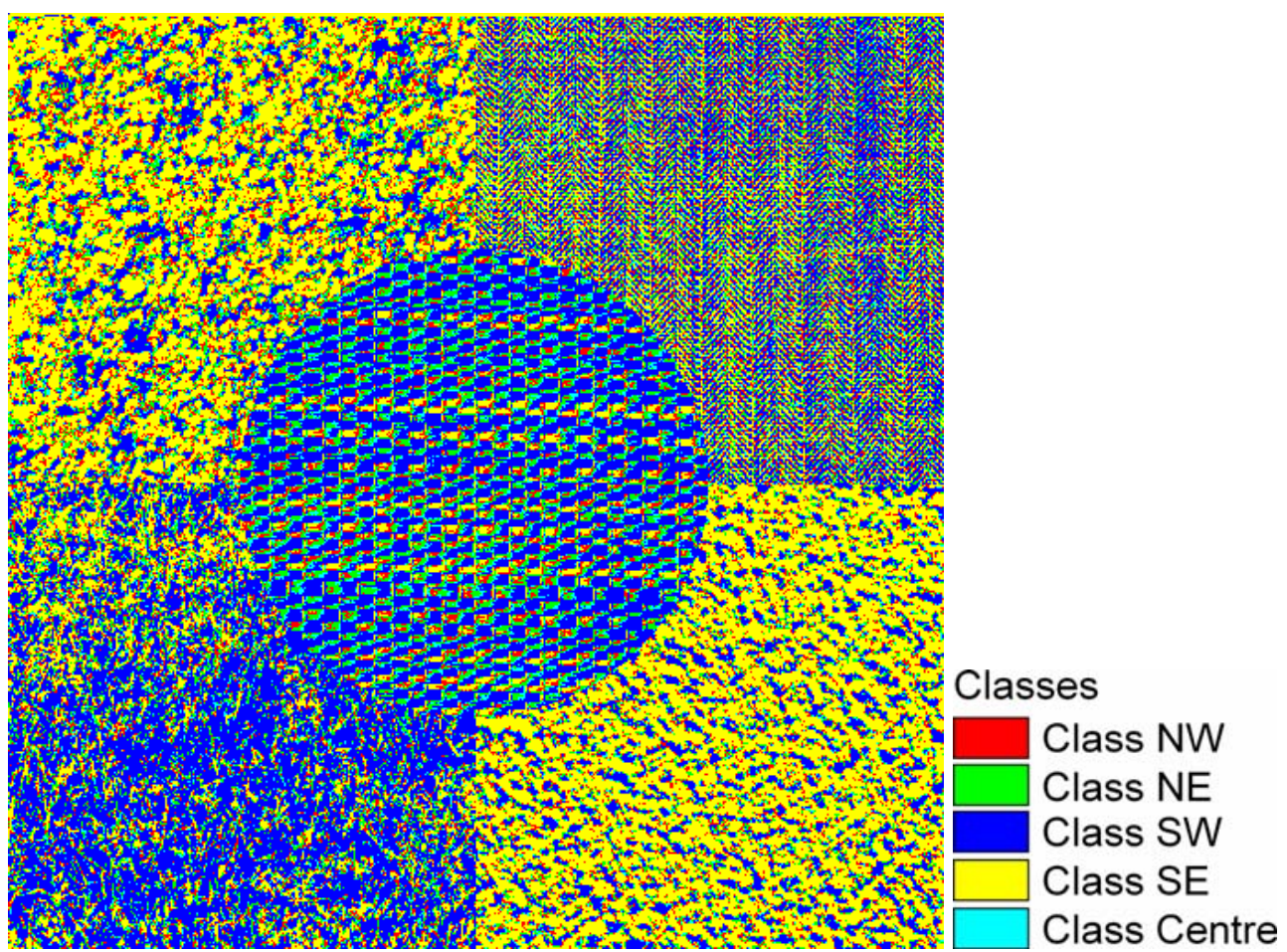

(b) 
Figure 2

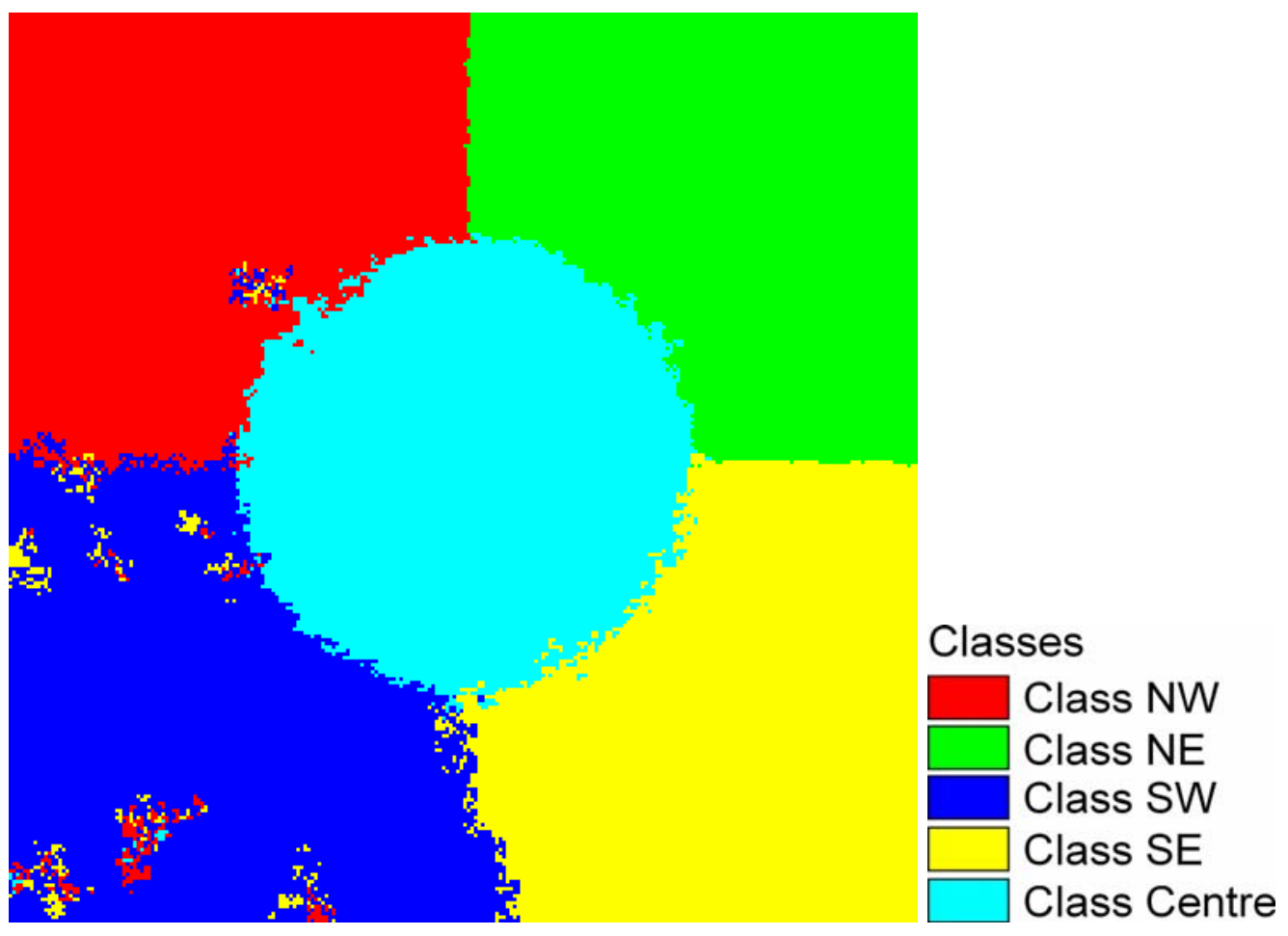

(a)

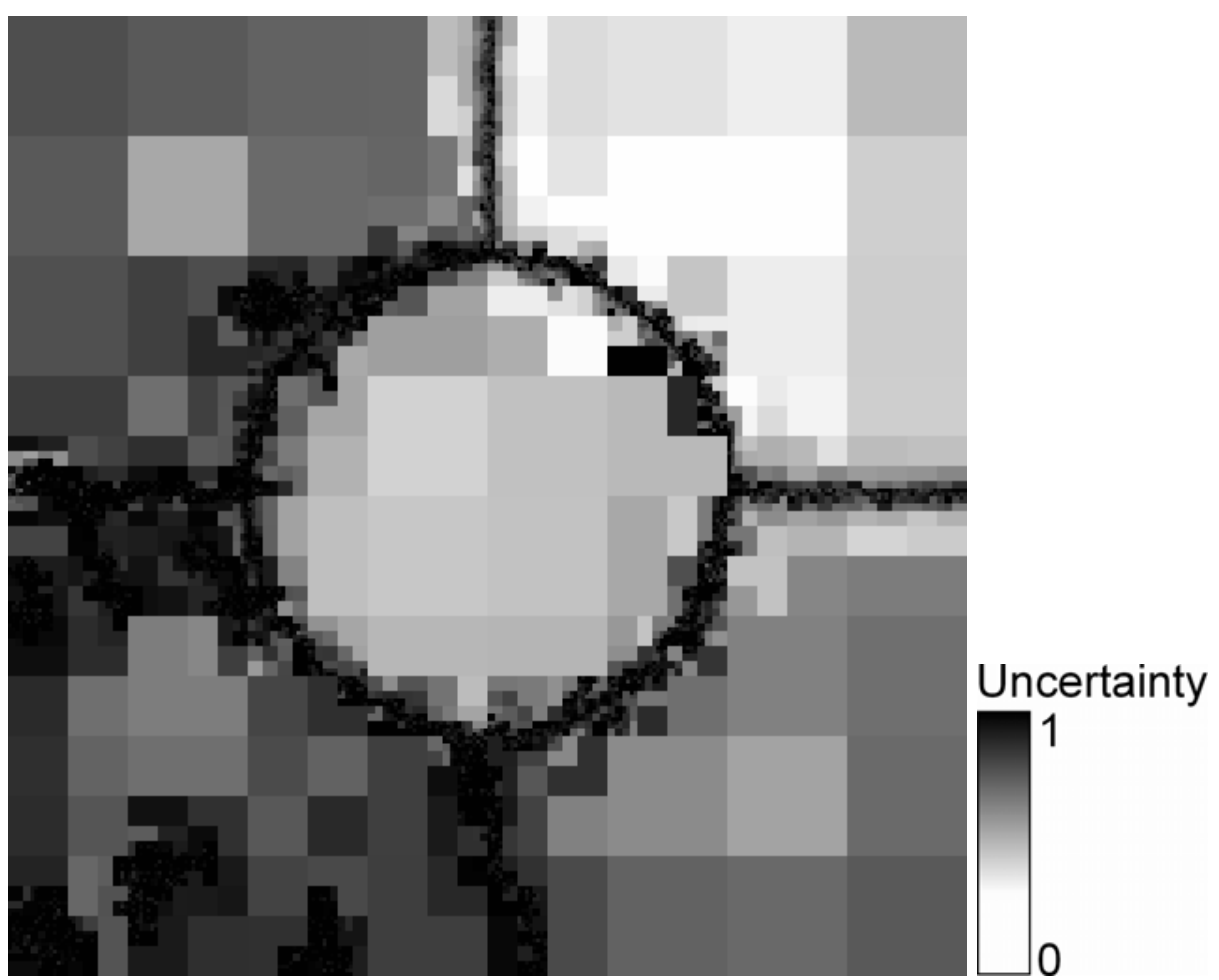

(b) 
Figure 3

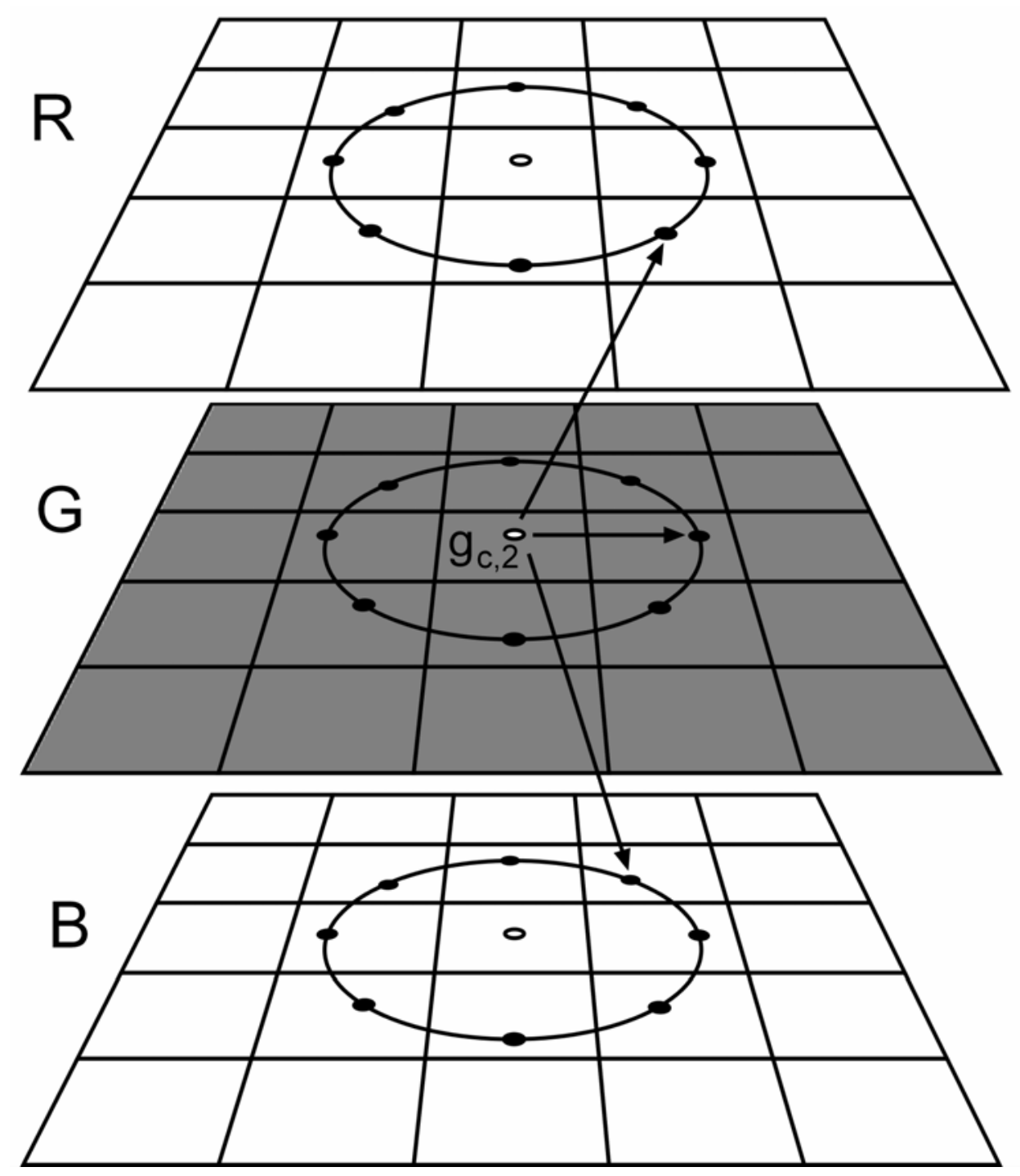


Figure 4

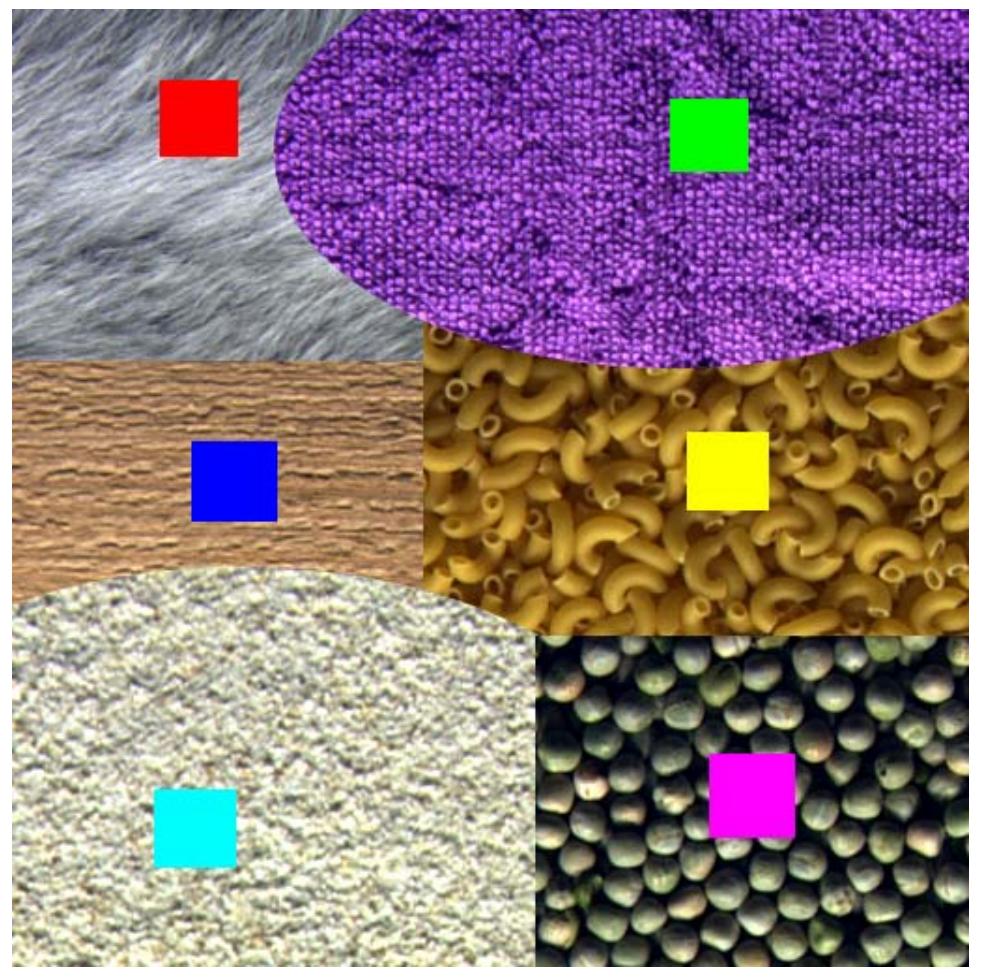

(a)

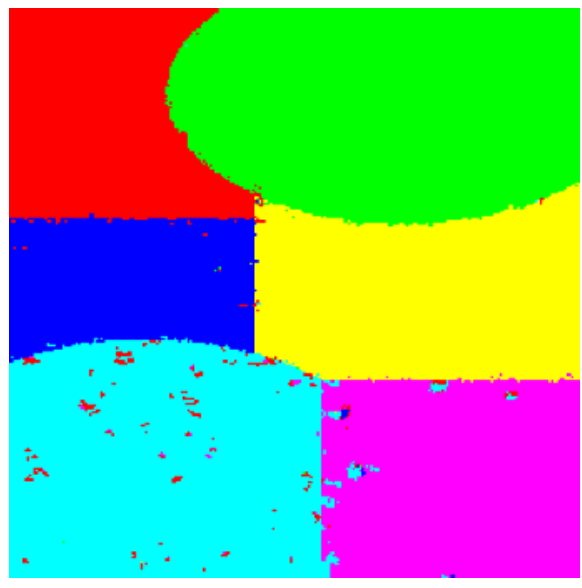
(b)
Lower Righ

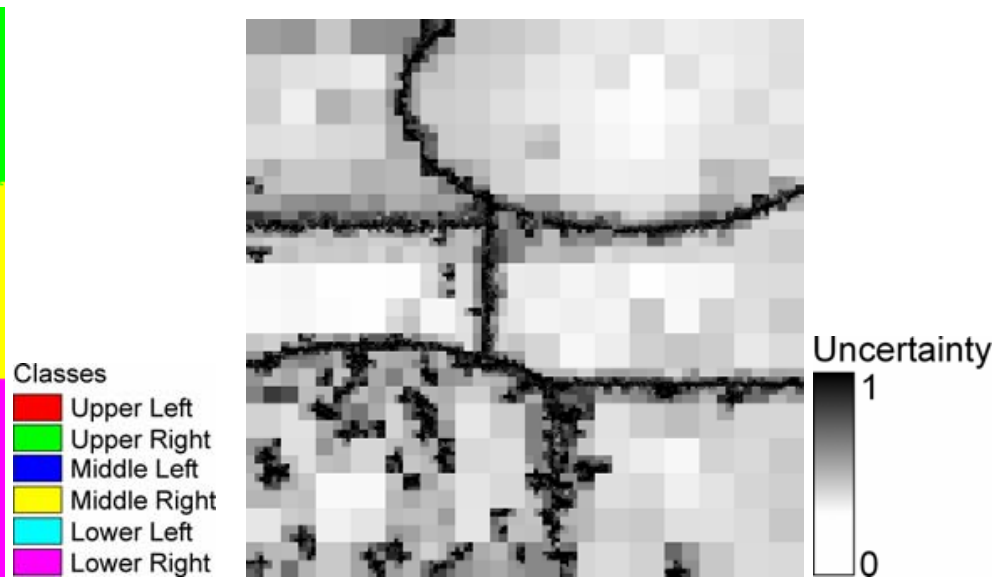

(c) 
Figure 5

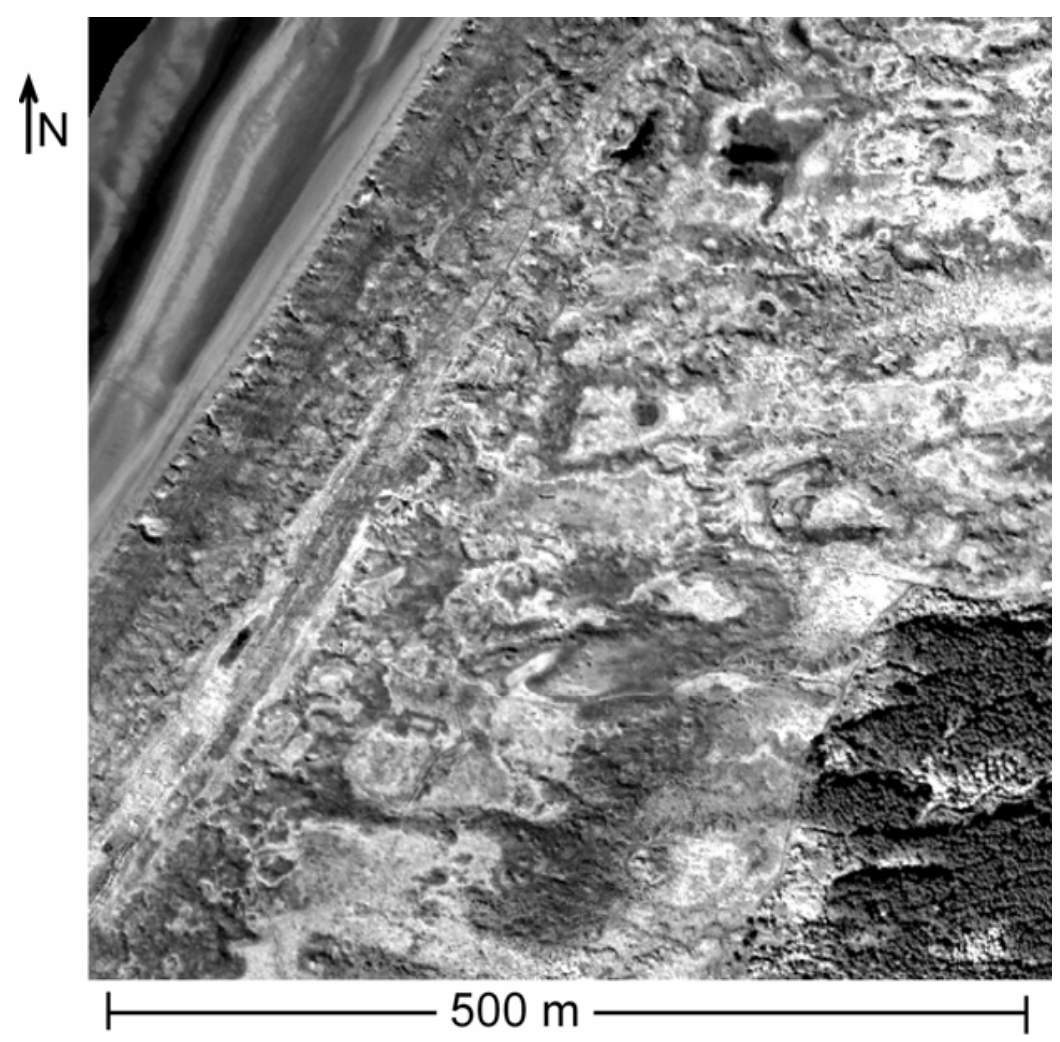

(a)

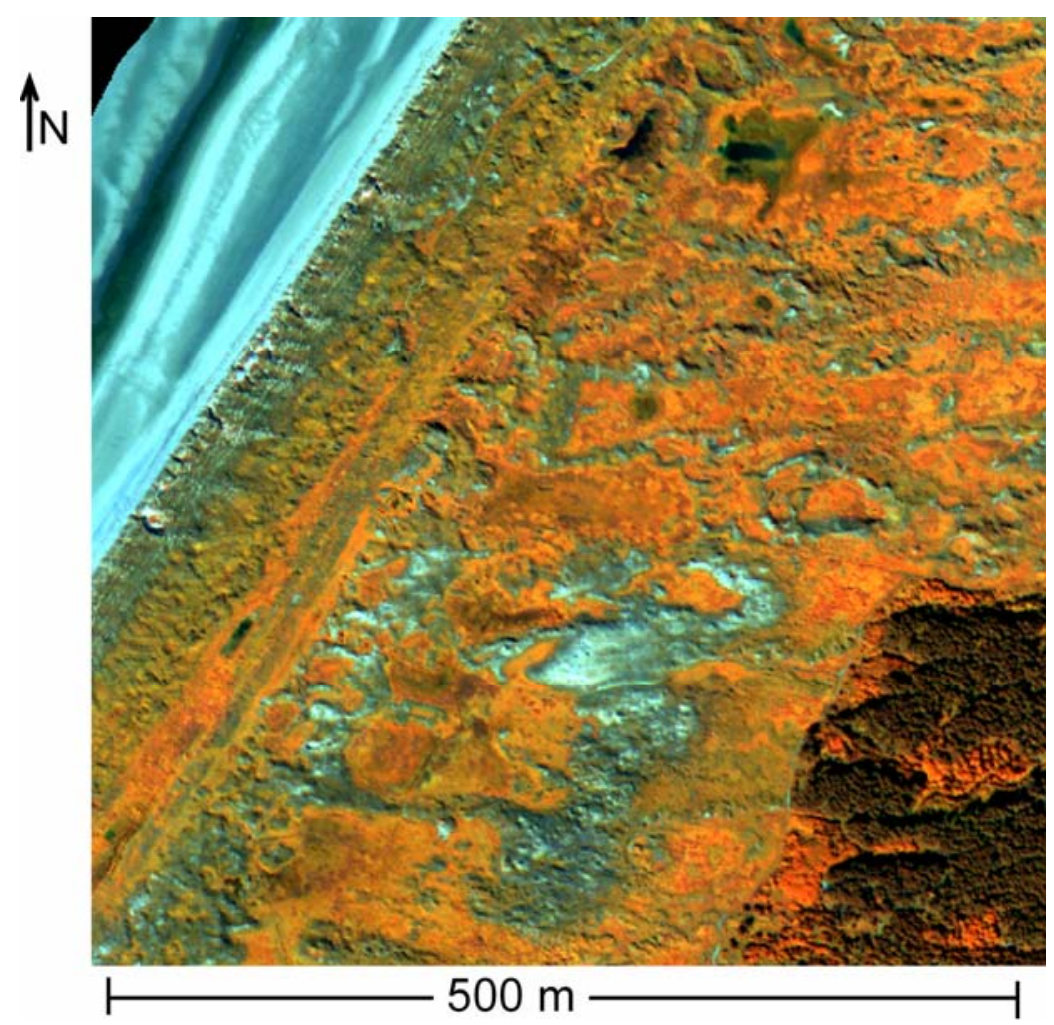

(b) 
Figure 6

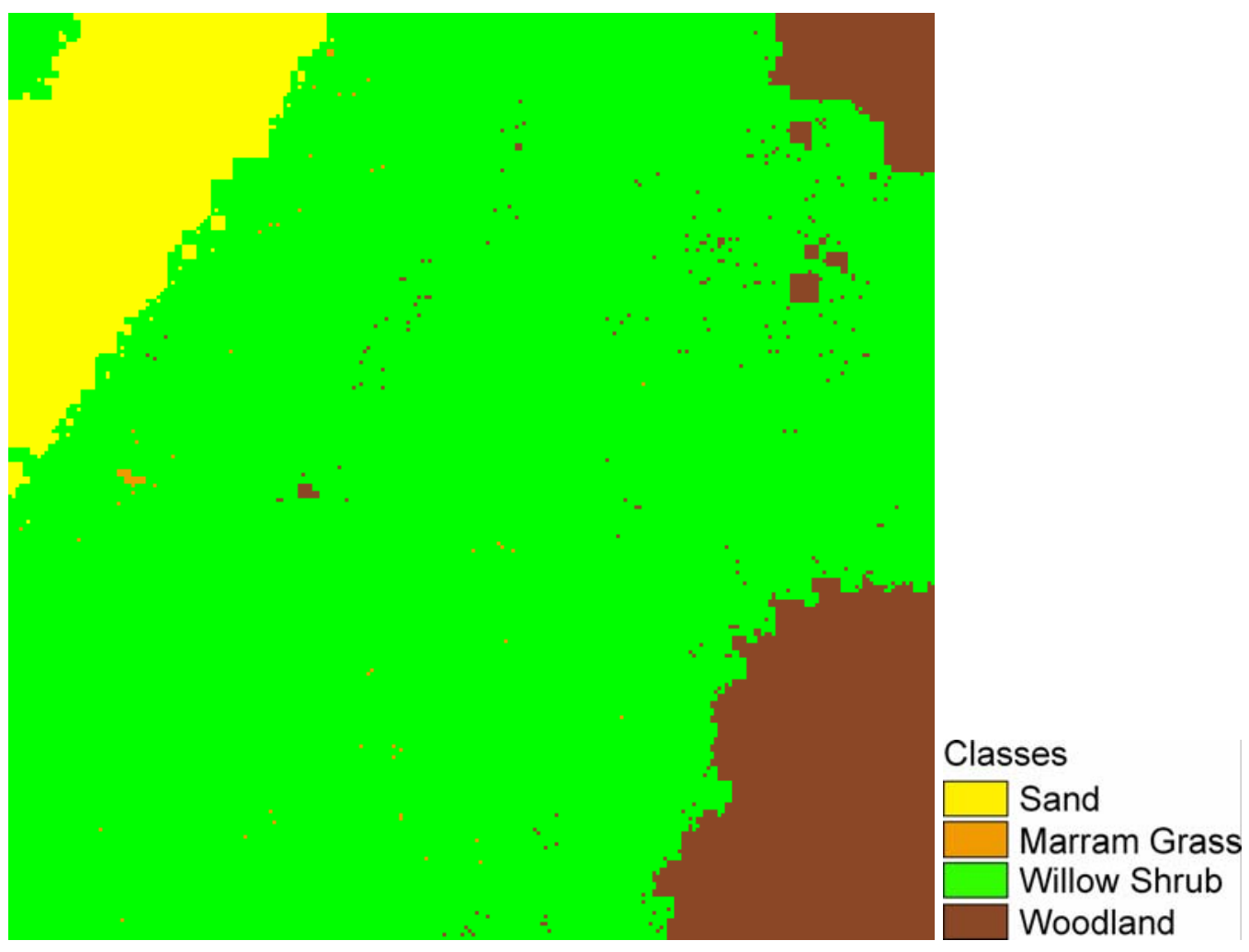

(a)

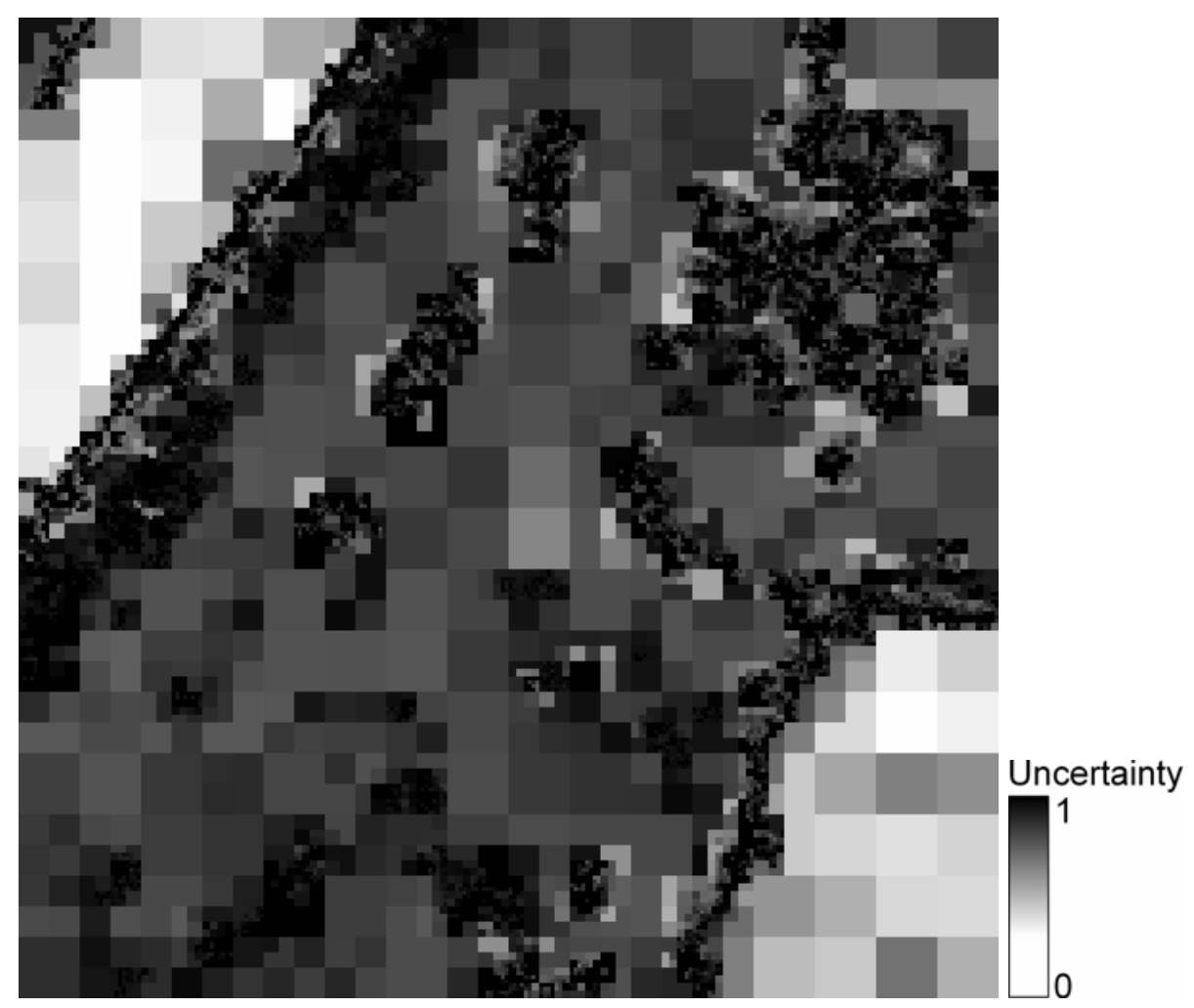

(b) 
Figure 7

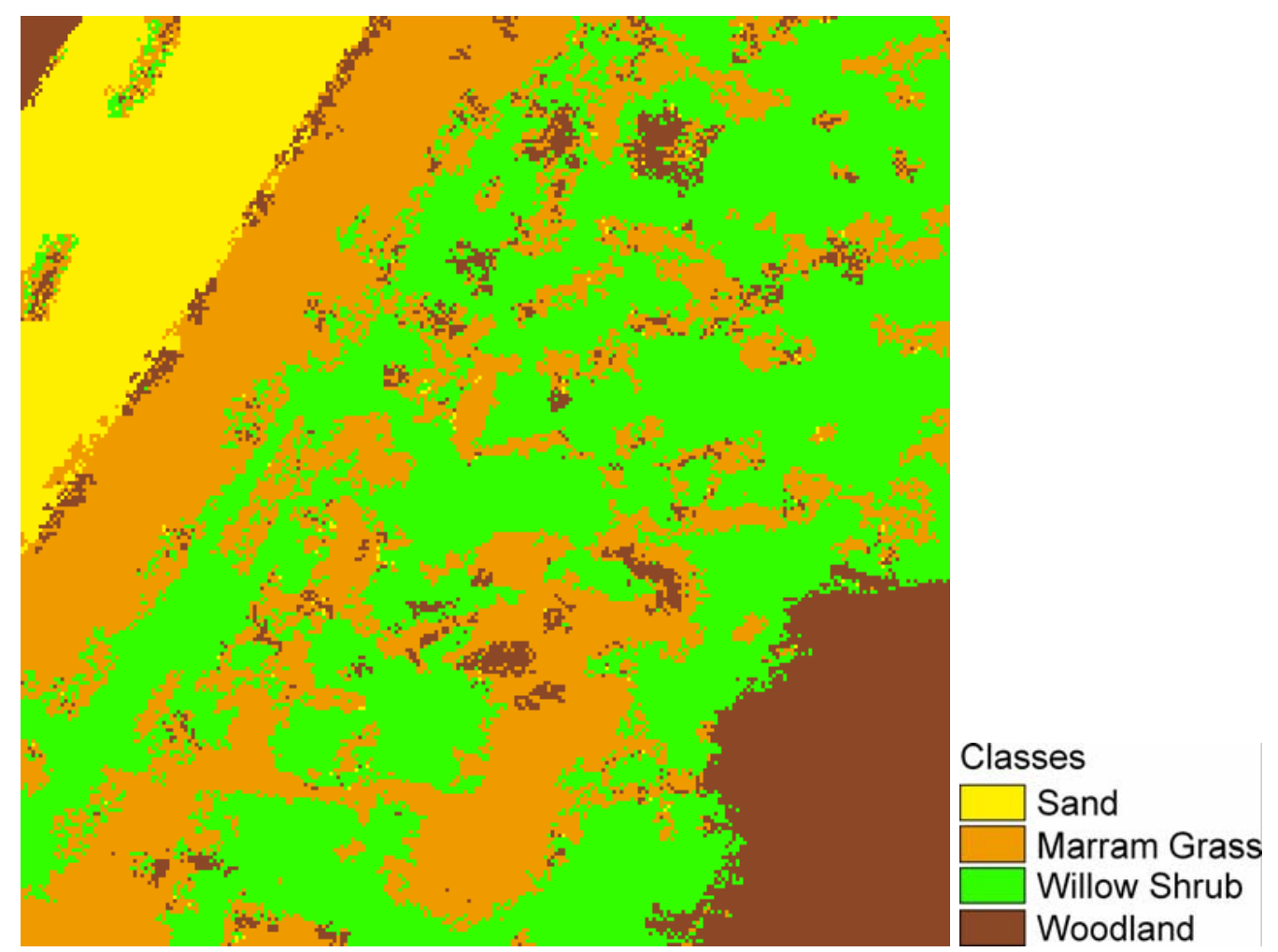

(a)

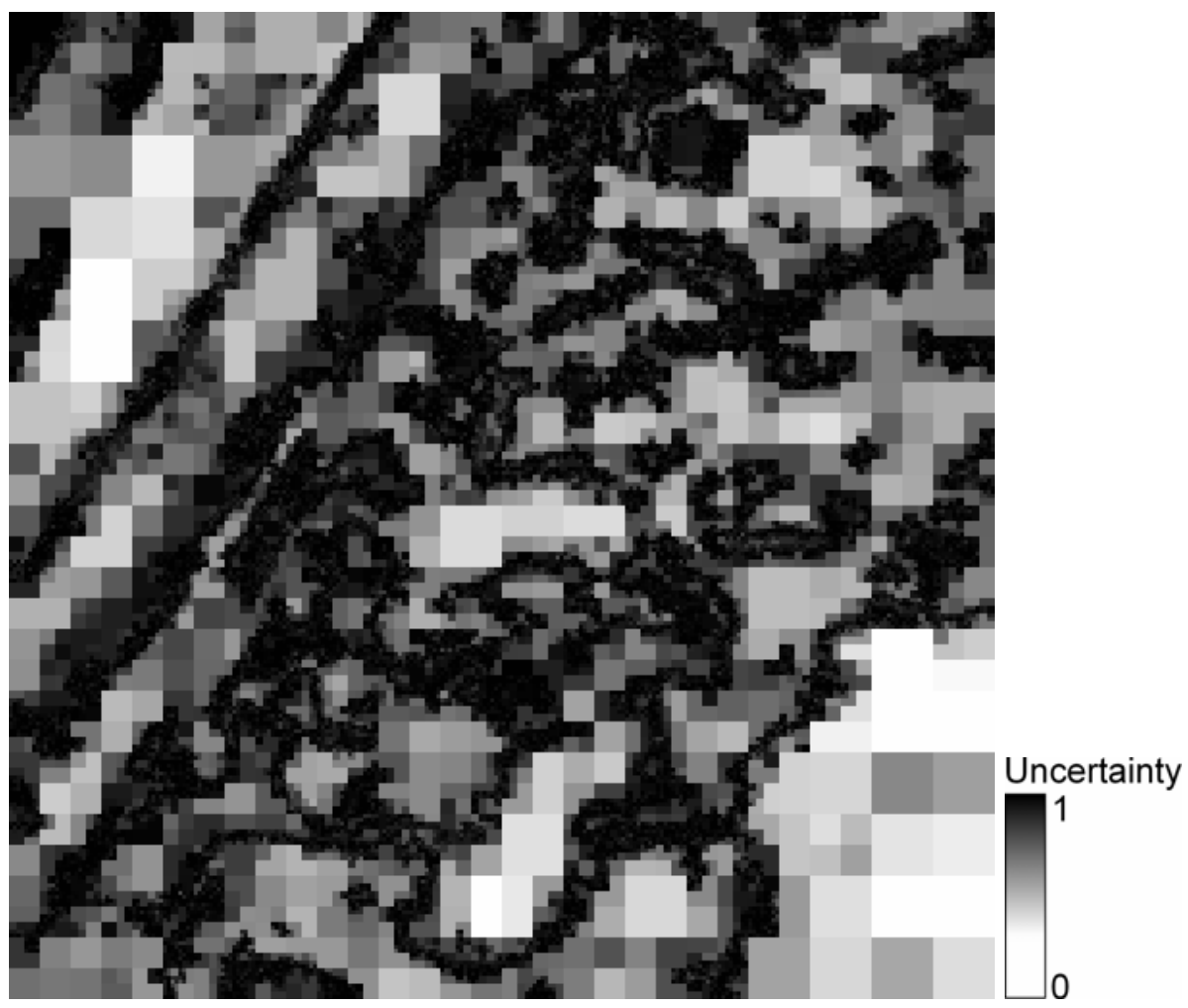

(b) 